\title{
Réponse des Instituts de recherche en santé du Canada à l'égard de la résistance
} aux antimicrobiens

\author{
Pagé $\mathrm{EL}^{1}$, Desnoyers $\mathrm{S}^{1}$, Létourneau $\mathrm{IJ}^{1}$, Keown $\mathrm{K}^{2}$, Jackson $\mathrm{A}^{2}$, Ouellette $\mathrm{M}^{1_{\text {* }}}$ \\ ${ }^{1}$ Instituts de recherche en santé du Canada, Institut des maladies infectieuses et immunitaires, Québec (Québec) \\ ${ }^{2}$ Instituts de recherche en santé du Canada, Ottawa (Ontario) \\ *Correspondance : $\underline{\text { marc.ouellette@crchul.ulaval.ca }}$
}

\section{Résumé}

La résistance aux antimicrobiens a été une priorité de recherche pour l'Institut des maladies infectieuses et immunitaires (IMII), des Instituts de recherche en santé du Canada (IRSC), depuis sa création, et de nombreuses initiatives de recherche stratégique ont été lancées pour régler ce problème de santé mondial en encourageant et en appuyant la recherche de mécanismes et de processus qui influent sur l'émergence et la propagation de la résistance chez les personnes et dans l'environnement. Nous présenterons ici des initiatives de recherche sur la résistance aux antimicrobiens menées par l'IMII des IRSC, qui comprennent des programmes nationaux ainsi que des partenariats internationaux avec le Royaume-Uni et l'Union européenne. Ces initiatives donnent d'ailleurs des résultats intéressants.

\section{Introduction}

Les Instituts de recherche en santé du Canada (IRSC) sont l'organisme du gouvernement fédéral responsable de l'investissement dans la recherche en santé. L'Institut des maladies infectieuses et immunitaires (IMII) est l'un des treize instituts « virtuels » des IRSC qui appuie la recherche et renforce les capacités de recherche dans le domaine des maladies infectieuses et de l'immunité, dans le cadre d'une approche fondée sur quatre grands thèmes (recherche biomédicale, recherche clinique, recherche sur les services liés aux systèmes de santé, et recherche sur la santé des populations, ses dimensions sociales et culturelles, et les influences environnementales sur la santé). Selon son plan stratégique de 2013-2018 (1), I'IMII des IRSC a élaboré des initiatives de recherche stratégique visant à stimuler les domaines ciblés, y compris la résistance antimicrobienne.

\section{Investissements des IRSC dans la recherche sur la résistance aux antimicrobiens et plan d’action fédéral sur la résistance aux antimicrobiens}

La résistance aux antimicrobiens est une menace croissante pour la santé publique qui est liée à une interaction entre de multiples secteurs. La perte d'antimicrobiens efficaces affaiblit la capacité de prévenir et de traiter les maladies infectieuses, tout en ayant une incidence sur notre système de soins de santé, le commerce mondial, l'agriculture, l'environnement et les secteurs de la santé. L'Organisation mondiale de la Santé a maintenant ajouté la résistance aux antimicrobiens aux problèmes de santé publique les plus pressants. En octobre 2014, le gouvernement du Canada a publié le document Résistance aux antimicrobiens et utilisation de ces derniers au Canada : un cadre d'action fédéral (2) afin qu'il serve de point de départ à une immobilisation et un engagement cohérents de toutes les personnes responsables d'agir relativement à la résistance aux antimicrobiens et l'utilisation de ces derniers. Les efforts sont concentrés sur trois domaines prioritaires : surveillance, intendance et innovation. Dans le cadre de leurs programmes ouverts et stratégiques, les IRSC ont investi 93,8 millions de dollars dans la recherche sur la résistance aux antimicrobiens entre 2009-2010 et 2013-2014, notamment plus de 15 millions de dollars en 2013-2014 seulement. La plupart des fonds étaient orientés vers l'innovation. À l'avenir, ces programmes pourront aider à mieux déterminer les stratégies et les approches visant l'intendance ou la surveillance. 


\section{Initiatives de recherche stratégique de l'IMII des IRSC sur la résistance aux antimicrobiens}

\section{Résistance aux antimicrobiens, répercussions sur le système de santé et résultats en matière de santé}

Durant la consultation avec la communauté de la recherche aux fins de notre plan stratégique de 2013-2018 pour l'IMII, la résistance aux antimicrobiens a clairement été cernée comme étant une priorité de recherche stratégique critique. Cela représente un continuum pour l'IMII, étant donné que l'Institut investit dans la recherche sur la résistance aux antimicrobiens depuis sa création. Des investissements antérieurs comprenaient des demandes liées à une initiative sur la Résistance aux antimicrobiens, aux répercussions sur le système de santé et aux résultats en matière de santé, ce qui a entraîné le financement de deux projets. Le premier projet financé a porté sur la résistance aux antimicrobiens chez les aînés et leur utilisation, et a étudié l'incidence des infections à Staphylococcus aureus résistant à la méthicilline (SARM) chez les patients âgés qui sont hospitalisés. L'équipe a montré que l'épidémiologie et les caractéristiques cliniques de ces patients (plus susceptibles d'être colonisés par les infections à SARM) sont différentes de celles des patients plus jeunes (3). Le second projet, appelé « Community-Acquired Antimicrobial Resistant Bacteria in Northern Canadian Communities », a mené à la création du « Northern Antibiotic Resistance Partnership », une équipe composée de membres de la collectivité, de professionnels de la santé, d'éducateurs et de chercheurs scientifiques qui étudient la résistance aux bactéries dans les collectivités du Nord (4). Le groupe a mis en place un programme de surveillance pour faire un suivi des infections bactériennes et de l'utilisation des antibiotiques, a élaboré des outils d'apprentissage pour les fournisseurs de soins de santé et la population en général, et a créé une étude cas-témoin pour déterminer les facteurs de risque associés aux infections à SARM acquises dans la communauté (SARM-AC) dans les régions du Nord de la Saskatchewan.

\section{Initiative de recherche sur la salubrité des aliments et de l'eau}

L'initiative de recherche sur la salubrité des aliments et de l'eau, qui a été en partie lancée à la suite à la tragédie survenue à Walkerton, a rassemblé des organismes gouvernementaux et privés pour élaborer une stratégie de recherche nationale visant à prévenir et à soigner les maladies causées par des agents pathogènes d'origine alimentaire et hydrique et les toxines connexes. Les deux appels de subventions ont entraîné le financement de quatre projets liés à la résistance aux antimicrobiens - un investissement de 2,8 millions de dollars. Les résultats de cet investissement sont notamment l'établissement de profils de résistance de base et les mécanismes de résistance observés avec les infections à Campylobacter jejuni chez la volaille de l'Alberta (5). Cet investissement a aussi permis la caractérisation des souches d'Escherichia coli résistant à la céfoxitine, ainsi que des mesures de prévention pour éviter la contamination bactérienne des cours d'eau $(6,7)$.

\section{Initiative de nouvelles solutions de rechange aux antibiotiques}

L'initiative de nouvelles solutions de rechange aux antibiotiques a été élaborée pour améliorer le financement existant offert dans le cadre des concours ouverts des IRSC en sollicitant des demandes axées sur de nouvelles approches en matière de résistance aux antibiotiques pour lesquelles le Canada avait peu ou pas de capacité de recherche. Trois thèmes prioritaires ont été déterminés : le système immunitaire; la phagothérapie (ou utilisation de virus pour infecter et tuer des bactéries pathogéniques); les systèmes physiques et les biomatériaux. L'investissement était le produit de la collaboration de plusieurs partenaires des secteurs public et privé. Par ailleurs, les projets financés ont engendré des résultats intéressants, y compris des résultats prometteurs découlant d'une technique non invasive de phagothérapie au moyen d'aérosols pour traiter l'infection des voies respiratoires (8) et de l'identification de nouveaux antibiotiques contre les infections à SARM. Suite à ce dernier projet, plusieurs brevets ont été déposés et la propriété intellectuelle a été transférée à une société créée conséquemment (9). 
Partenariat Canada-Royaume-Uni sur la résistance aux antimicrobiens Depuis 2007, le Canada travaille en partenariat avec le Medical Research Council (MRC) du Royaume-Uni, ce qui a entraîné plusieurs ateliers et concours conjoints. Après un premier atelier et un financement de départ, en septembre 2010, une subvention d'équipe visant un partenariat entre le Canada et le Royaume-Uni sur la résistance aux antibiotiques a été offerte pour travailler à partir de collaborations existantes entre les deux pays, et ce, pour 4 ans. La première équipe financée a travaillé sur la synthèse de la paroi cellulaire des bactéries et met en ce moment à l'essai plusieurs inhibiteurs potentiels, afin de trouver des pistes pour créer de nouveaux antibiotiques (10-12). La deuxième équipe a étudié la résistance bactérienne aux antimicrobiens de la classe des $\beta$-lactamines, ce qui a donné de nombreux résultats, y compris la conception, la synthèse et l'essai de nouveaux candidats inhibiteurs de métallo- $\beta$-lactamases, et la caractérisation de l'inhibition de cibles clés liées aux métallo- $\beta$-lactamases par des composantes connues $(13,14)$. L'investissement dans le cadre du partenariat Canada-Royaume-Uni était de près de 4 millions de dollars et a permis aux équipes financées d'obtenir des fonds supplémentaires et de participer à d'autres partenariats, ainsi que de renforcer la capacité dans les deux domaines. Un second atelier, portant sur les stratégies translationnelles visant à lutter contre la résistance aux antibiotiques, a ensuite été présenté en 2013. Cet atelier a permis de fournir des recommandations pour accroître la sensibilisation, pour appuyer les partenariats des secteurs public et privé, pour hausser les investissements financiers et soutenir les approches collaboratives (15).

\section{Initiative de programmation conjointe sur la résistance aux antimicrobiens}

Le Canada, par l'entremise des IRSC, est un important bailleur de fonds de l'Initiative de programmation conjointe sur la résistance aux antimicrobiens (IPCRAM), un réseau international de 20 pays fournissant une plate-forme de collaboration pour que l'on passe de la sensibilisation à l'action en ce qui concerne la résistance aux antimicrobiens en appuyant la recherche et en facilitant son application dans l'industrie et les politiques. Le but de cette initiative est d'élaborer des approches intégrées afin d'en arriver à une recherche unique sur la résistance aux antimicrobiens à l'échelle mondiale. Grâce à son programme de recherche stratégique, I'IPCRAM renforce la collaboration multidisciplinaire et permet d'assurer que les lacunes liées aux connaissances sont rapidement cernées et comblées. Les mesures prises dans six sujets prioritaires (thérapie, diagnostic, surveillance, transmission, environnement et interventions) formeront de nouvelles stratégies de prévention et d'intervention afin d'améliorer la santé publique et d'offrir des avantages économiques et sociétaux. Lancé en 2014, l'appel transnational InnovaRésistance : approches novatrices visant à traiter la question de la résistance aux antibactériens a entraîné le financement de sept consortiums, dont six comprenaient des chercheurs canadiens (16). Le but premier de cet appel conjoint est de combiner les ressources, les infrastructures et l'expertise de recherche de pays multiples afin de combattre la résistance aux antibiotiques. L'appel était axé sur la réévaluation de composés antimicrobiens existants (seul ou en combinaison avec d'autres médicaments, modulateurs immunitaires ou approches antibactériennes), l'identification de nouvelles cibles bactériennes ou de nouveaux composés thérapeutiques, la découverte de nouveaux traitements pour lutter contre les mécanismes connus de résistance aux antimicrobiens et pour restaurer la sensibilité aux antibiotiques ou aux combinaisons de médicaments traditionnels, et les stratégies visant à inhiber ou à réduire l'acquisition d'une résistance. Les projets canadiens nominés touchaient plusieurs de ces priorités.

Récemment, l'IPCRAM a annoncé un autre appel transnational, qui sera lancé au début de 2016, auquel participeront 20 organismes de financement partout dans le monde, y compris au Canada. Le sujet de cet appel encouragera la recherche visant à « éclaircir la dynamique de transmission et de sélection de la résistance aux antimicrobiens tant aux niveaux génétique, bactérien, animal, humain, social et environnemental, afin de concevoir et d'évaluer les mesures de prévention et d'intervention pour contrôler la résistance » (17).

L'IPCRAM est vouée à l'approche « Une santé », selon laquelle des collaborations multidisciplinaires sont créées pour s'attaquer aux questions de soins de santé. Par conséquent, la recherche sur les mécanismes menant à la propagation de la résistance dans les réservoirs et entre ces derniers, y compris les animaux, l'environnement et les gens, contribuera à la conception de mesures préventives pour contrer la menace de la résistance aux antimicrobiens envers la santé publique. 


\section{Autres investissements stratégiques des IRSC}

Relativement à l'approche « Une santé », les IRSC ont mis en place l'initiative phare Environnements et santé, qui intègre une approche axée sur les domaines prioritaires connexes (trois domaines prioritaires connexes) au moyen de l'étude d'expositions environnementales cumulatives, et de leurs interconnexions, leurs intersections et leurs répercussions sur la santé et la maladie tout au long de la vie (18). L'appel transnational de l'IPCRAM à venir en 2016 cadre avec les sujets de l'initiative Environnements et santé. Les deux initiatives appuieront la recherche visant à examiner la façon dont les stratégies et les approches intersectorielles aident à améliorer la santé de la population et l'équité en matière de santé.

\section{Conclusion}

La résistance aux antimicrobiens est un problème sérieux qui cause l'hospitalisation de plus de 250000 Canadiens chaque année, dont plus de 18000 de ces patients hospitalisés contractent des infections résistantes aux médicaments (19). Par exemple, les décès directement liés à la bactérie Clostridium difficile ont à eux seuls quintuplé au cours de la dernière décennie (20). La résistance aux antimicrobiens représente également un fardeau financier important. Selon une recherche initiale, si aucune mesure n'est prise, une hausse continue de la résistance entraînerait, en 2050, 10 millions de décès et un coût de 100 mille milliards de dollars américains à l'échelle mondiale (21). L'IMII des IRSC est engagé à améliorer la vie des Canadiens et de la collectivité mondiale en appuyant la recherche novatrice sur la résistance aux antimicrobiens. Au cours des cinq dernières années, l'IMII et les IRSC ont investi plus de 93,8 millions de dollars dans la lutte contre la résistance aux antimicrobiens. La recherche sur le sujet demeurera une priorité puisque la menace continue de grandir. Les IRSC et l'IMII jouent un rôle proactif dans le plan d'action fédéral sur la résistance aux antimicrobiens et continueront d'encourager l'innovation en appuyant des collaborations nationales et internationales et des initiatives à thèmes multiples afin de stimuler les investissements. Les investissements futurs dans la recherche stratégique seront liés à la création de nouvelles molécules, mais aussi à des stratégies visant à protéger les antibiotiques actuels grâce à un diagnostic et une intendance améliorés. Il existe actuellement un élan dans le domaine de la recherche et des politiques vers la recherche de solutions novatrices pour attaquer le problème épineux de la résistance aux antimicrobiens, qui a été qualifié dans le cadre du Forum économique mondial de 2013 comme étant le plus grand risque de complaisance pouvant nuire à la santé humaine.

\section{Remerciements}

Les auteurs souhaitent remercier l'équipe d'administration précédente de l'IMII des IRSC, sous la direction du $D^{r}$ Bhagirath Singh, pour son travail acharné relativement aux programmes sur la résistance aux antimicrobiens. Ils remercient également tout particulièrement l'équipe qui travaille sur la résistance aux antimicrobiens aux IRSC.

\section{Conflit d'intérêts}

Aucun 


\section{Références}

(1) Canadian Institutes of Health Research. Institutes-Infection and Immunity. III Strategic Plan 2013-18. 2013 Dec. (Disponible en français : http://www.cihr-irsc.gc.ca/f/46554.html

(2) Government of Canada. Antimicrobial Resistance and Use in Canada: A Federal Framework for Action. Ottawa, ON: Public Healty Agency of Canada; 2014 Oct. (Disponible en français :

http://healthycanadians.gc.ca/alt/pdf/drugs-products-medicaments-produits/buying-using-achatutilisation/antibiotic-resistance-antibiotique/antimicrobial-framework-cadre-antimicrobiens-fra.pdf)

(3) Simor AE, Ofner-Agostini M, Paton S, McGeer A, Loeb M, Bryce E, et al. Clinical and epidemiologic features of methicillin-resistant Staphylococcus aureus in elderly hospitalized patients. Infect Control Hosp Epidemiol. 2005 Oct;26(10):838-41.

(4) Northern Antibiotic Resistance Partnership (NARP). http://www.narp.ca/

(5) Kos VN, Keelan M, Taylor DE. Antimicrobial susceptibilities of Campylobacter jejuni isolates from poultry from Alberta, Canada. Antimicrob Agents Chemother. 2006 Feb;50(2):778-80.

(6) Mataseje LF, Neumann N, Crago B, Baudry P, Zhanel GG, Louie M, et al. Characterization of cefoxitin-resistant Escherichia coli isolates from recreational beaches and private drinking water in Canada between 2004 and 2006. Antimicrob Agents Chemother. 2009 Jul;53(7):3126-30. doi: 10.1128/AAC.01353-08. Epub 2009 Apr 27.

(7) Zhu Z, Broersma K, Mazumder A. Model assessment of cattle and climate impacts on stream fecal coliform pollution in the Salmon River watershed, British Columbia, Canada. Water Air Soil Pollut. 2011;215:155-76. doi: 10.1007/s11270-010-0467-0.

(8) Semler DD, Goudie AD, Finlay WH, Dennis JJ. Aerosol phage therapy efficacy in Burkholderia cepacia complex respiratory infections. Antimicrob Agents Chemother. 2014 Jul;58(7):4005-13. doi: 10.1128/AAC.02388-13. Epub 2014 May 5. DeNovaMed. http://www.denovamed.com

(9) Farha MA, Czarny TL, Myers CL, Worrall LJ, French S, Conrady DG, et al. Antagonism screen for inhibitors of bacterial cell wall biogenesis uncovers an inhibitor of undecaprenyl diphosphate synthase. Proc Natl Acad Sci USA. 2015 Sep 1;112(35):11048-53. doi: 10.1073/pnas.1511751112. Epub 2015 Aug 17.

(10) Kouidmi I, Levesque RC, Paradis-Bleau C. The biology of Mur ligases as an antibacterial target. Mol Microbiol. 2014 Oct;94(2):242-53. doi: 10.1111/mmi.12758. Epub 2014 Sep 5.

(11) Czarny TL, Perri AL, French S, Brown ED. Discovery of novel cell wall-active compounds using P ywaC, a sensitive reporter of cell wall stress, in the model gram-positive bacterium Bacillus subtilis. Antimicrob Agents Chemother. 2014 Jun;58(6):3261-9. doi: 10.1128/AAC.02352-14. Epub 2014 Mar 31.

(12) Ghavami A, Labbé G, Brem J, Goodfellow VJ, Marrone L, Tanner CA, et al. Assay for drug discovery: Synthesis and testing of nitrocefin analogues for use as $\beta$-lactamase substrates. Anal Biochem. 2015 Oct 1;486:75-7. doi: 10.1016/j.ab.2015.06.032. Epub 2015 Jul 2.

(13) Rotondo CM, Marrone L, Goodfellow VJ, Ghavami A, Labbé G, Spencer J, et al. Arginine-containing peptides as potent inhibitors of VIM-2 metallo- $\beta$-lactamase. Biochim Biophys Acta. 2015 Nov;1850(11):2228-38. doi: 10.1016/j.bbagen.2015.07.012. Epub 2015 Aug 1.

(14) Canadian Institutes of Health Research (CIHR). Translational Strategies to Combat Antibiotic Resistance: A Call to Action-Workshop Report. A Canada/UK Collaboration between: Canadian Institutes of Health Research, Institute of Infection and Immunity, UK Health Protection Agency, Canadian High Commission. Canada House, London, UK. 2013 Feb 6-7. Ottawa, ON: CIHR; 2014. (Disponible en français : http://www.cihrirsc.gc.ca/f/48215.html)

(15) Joint Programming Initiative on Antimicrobial Resistance (JPIAMR). Projects. Results from the first JPIAMR transnational call InnovaResistance: Innovative approaches to address antibacterial resistance. 2014. http://www.jpiamr.eu/wp-content/uploads/2014/12/Results-from-the-first-transnational-callInnovaResistance1.pdf

(16) Joint Programming Initiative on Antimicrobial Resistance (JPIAMR). News. Save the date: Transmission dynamics call for proposals to open in January 2016. 2015 Jul 23.

http://www.jpiamr.eu/save-the-date-transmission-dynamics-call-for-proposals-to-open-in-january-2016/

(17) Canadian Institutes of Health Research. Initiatives. Environments and Health: Overview. 2015 Feb 19. (Disponible en français : http://www.cihr-irsc.gc.ca/f/48465.html)

(18) Zoutman DE, Ford BD, Bryce E, Gourdeau M, Hébert G, Henderson E, et al. The state of infection surveillance and control in Canadian acute care hospitals. Am J Infect Control. 2003 Aug;31(5):266-72; discussion 272-3.

(19) Gravel D, Miller M, Simor A, Taylor G, Gardam M, McGeer A, et al. Health care-associated Clostridium difficile infection in adults admitted to acute care hospitals in Canada: A Canadian Nosocomial Infection Surveillance Program study. Clin Infect Dis. 2009 Mar 1;48(5):568-76.

(20) O'Neill J. Antimicrobial Resistance: Tackling a crisis for the health and wealth of nations. The Review on Antimicrobial Resistance. 2014 Dec. http://amr-review.org/Publications 